\title{
A Cancer Survivorship Model from the Lens of Occupational Science Incorporating Patient Self Management - Model of Occupational Participation
}

\section{Loh Siew Yim}

Faculty of Medicine, University of Malaya, Kuala Lumpur, Malaysia

\begin{abstract}
Over the last few decades, several cancer survivorship models of care have evolved to address the needs of the rising population of cancer survivors worldwide. The various models of survivorship care have evolved from the perspective of cancer center, community setting, as well as country-wide health care system, as a way to address the unique, and heterogeneous needs of post treatment survivors. However, in line with the rationale survivors will require preventive and general supportive community care subsequent to their overall functioning and health-related care, a model of occupational participation in cancer survivorship is proposed, to empower survivors to work alongside the community they lived in for support and for advocating cancer control.
\end{abstract}

\author{
Publication History: \\ Received: October 08, 2020 \\ Accepted: December 09, 2020 \\ Published: December 11, 2020

\section{Keywords:} \\ Model of occupational \\ participation, Cancer survivorship
}

\section{Current Care Model}

People diagnosed with cancer spend significant time undergoing treatment where there are often major disruptions in their everyday life. Disrupted occupational engagements are caused by the loss of bodily part and/or functions, the loss of social roles, lowers a survivor's capacity to engage in basic daily activities. As cancer treatment is often prolonged and takes a minimum of several months to complete the combination of aggressive treatment which covers surgery, chemotherapy, and radiotherapy, these disrupted daily occupational activities must be re-engaged and not everyone has a successful return or reconnecting back to life.

There are many models of cancer survivorships $[1,2]$ that has been proposed but most of these models are still primarily focussing on provision of care and continuation of care. As an example, Loonen and collegues [2] proposed a "personalized cancer survivorship care model" which they claimed are adaptable for various healthcare settings. The model has a strong component of 'care' for survivors, with its two-part care modules i.e i) personalized follow-up care in line with person-centered-care, aiming to empower survivors, and ii) a multidisciplinary organisational approach with three components of initiating, integrating and safeguarding partnership with the patient [2]. Another model, the Memorial Sloan Kettering Cancer Center (MSKCC) is a longitudinal model which aim to cover the past, present, and future plans of 'care' within a single institution such as Memorial Sloan Kettering Cancer Center (MSKCC) and which was distinctively designed to address those smaller groups of high-risk survivors with multiple therapy-related comorbidities to benefit from specialized, follow-up care [1]. Yet another model, the Fort Worth Program for Community Survivorship (ProComS), was designed as a multidisciplinary community service aim to address the fragmented car. for oncology patients through an integrated community model, by providing ongoing supportive, psychosocial, and behavioral care for survivors, with oncology-certified registered nurse evaluations, social worker assessments, exercise training, registered dietitian consultations, and psychologic and certified genetic counselling, as well as a diagnosis-driven support groups with a variety of group learning opportunities. Evidently, most of these model are heavily infused with 'care' modules.

\section{Principles of Survivorship Care}

Survivorship care has been viewed as a specific approach to address the long-term needs at post-treatment phase, a need based care triggered by the 2005 Institute of Medicine (IOM) report (from Cancer Patient to Cancer Survivor: Lost in Transition), which delineated four key elements of care during the survivorship phase : i) prevention and detection of new cancers and recurrent cancer; ii) surveillance for recurrence or new primaries; iii) interventions for long-term and late effects (hereafter referred to as late effects) from cancer and its therapies; and iv) coordination between specialists and primary care providers to ensure that all of the survivor's needs are met. Most model of survivorship care address these core elements and as highlighted above, are heavily advocating survivors as another new generation that requires a transitional coverage of care as they move from patients to survivors. However, there is a proactive need to address the increasing number of rising survivors as a projected 1:2 people will be diagnosed with cancer in their lifetime. Hospitals resources are not able to address this exponential increase of survivors needing care, and if all these models are projecting the survivors as passive receivers, then, a new model to address this gap is needed. The rationale is shared partnership to care, active carer vs passive recipients, pensive and waiting for symptoms vs active pursuer of health and looking for wellbeing and health index.

\section{A Model Addressing the Gap to Re-Connect Back to Life's New Norms}

The Loh \& Jonsson's [3] model of occupational participation in cancer survivorship (MOPCS) was proposed to address the gap that care is not passive, but rather active and initiated by the survivors as active participants of their wellbeing and health. The concepts

"Corresponding Author: Prof. Dr Loh Siew Yim, Faculty of Medicine, University of Malaya, Kuala Lumpur, Malaysia; E-mail: syloh@um.edu.my

Citation: Yim LS (2020) A Cancer Survivorship Model from the Lens of Occupational Science Incorporating Patient Self Management - Model of Occupational Participation. Int J Psychol Behav Anal 6: 171. doi: https://doi. org/10.15344/2455-3867/2020/171

Copyright: (C) 2020 Yim. This is an open-access article distributed under the terms of the Creative Commons Attribution License, which permits unrestricted use, distribution, and reproduction in any medium, provided the original author and source are credited. 
Citation: Yim LS (2020) A Cancer Survivorship Model from the Lens of Occupational Science Incorporating Patient Self Management - Model of Occupational Participation. Int J Psychol Behav Anal 6: 173. doi: https://doi.org/10.15344/2455-3867/2020/173

Page 2 of 3

of monitoring (for) and managing long term and late effects will be monitored by the survivors (not healthcare practitioners) and in line with patient self management principle - survivors are the experts living with the condition. In addition, a greater role that demands survivors' participation is the role of social-preventive and health promotional tasks and these calls for survivors to take charge. However, many cancer survivorship model focus on passive care for the cancer survivors. Therefore, Loh and Jonsson [3] conceptualised the Occupational Model in Cancer Survivorship (MOPCS) from the perspective of occupational science, where the term 'occupation' denotes an act of doing ie active person, not a passive recipient. The MOPCS favoured and incorporated the Institute of medicine (2006)'s four components of survivorship care listed above, but, it illustrated these tasks as active occupational participation tasks and comes under the concept o. 'resuming health after cancer' -tasks to be participated actively be survivors. (Figure 1). At this phase, 'resuming' means readjusting and reconnecting back either using a new norm or the previous norm, with adjustment and a goal towards a healthier lifestyle. The rationale behind this MOPCS model is that the act of doing or work is needed for prevention of recurrences, cancer control but also prevention of other comorbidities and strengthening all key aspects of physical, mental and occupational health, to ensure survivors can keep well.
The MOPCS model has a vertical theme that runs across the four Institute of Medicine's components, as the unique 'occupational participation' theme - i.e the essential core element of 'occupation' as the basis to better human performance. Loh \& Jonsson 's MOPCS Model [3] addresses the gap in the International Classification of Functioning (ICF)'s framework to emphasise the 'resuming to health' work that needs to be done through a partnership care approach with cancer survivors.

The 'personal' factors (e.g., key attribute like self efficacy act as the key tenet for successful behaviour change), and the environmental factors influencing adaptation after cancer (will needs to be assessed, monitored and modified). With this model which calls for active participation of survivors in the community, and which allows better working platform, together with society for cancer control activities. One of the barriers in cancer control campaigns is cancer stigma, and society must help to reduce stigma of cancer. Society must change their perception of viewing cancer as a fatal and life-threatening condition, thus creating a fear and causing unease and avoidances of the topic. Cancer is currently an ever-expanding presence in the society. Therefore, there is great benefit to reconciliate the ability of 'cancer' to scare and isolate people, with its positive ubiquity view of overcoming and living with cancer effectively. Destigmatising,

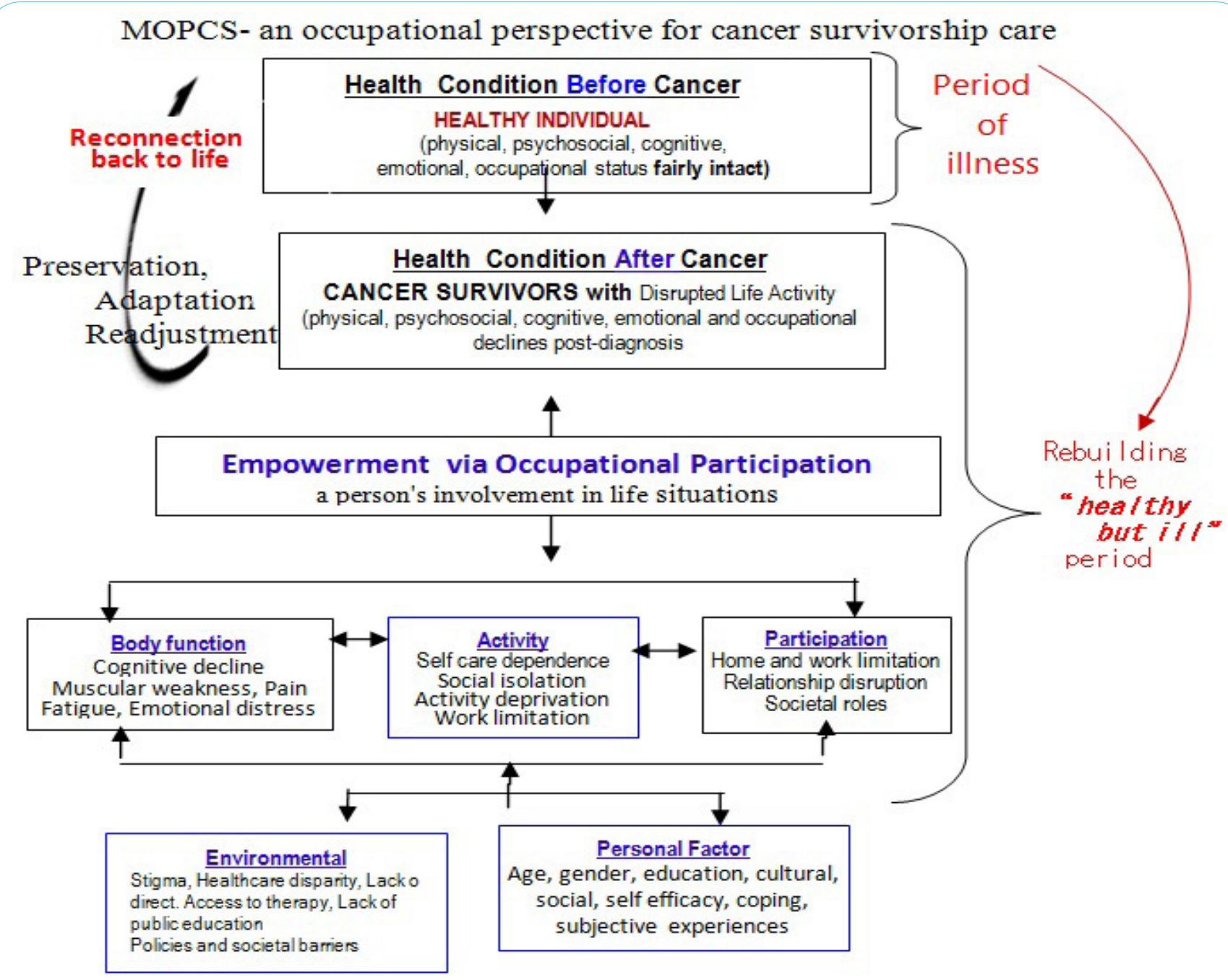

Figure 1: Model of Occupational-Participation in Cancer survivorship [MOPCS]. 
Citation: Yim LS (2020) A Cancer Survivorship Model from the Lens of Occupational Science Incorporating Patient Self Management - Model of Occupational Participation. Int J Psychol Behav Anal 6: 173. doi: https://doi.org/10.15344/2455-3867/2020/173

Page 3 of 3

desensitising issues of cancer in the society can be facilitated with an increasing focus on offering therapeutic (physical, occupational, social-preventive) intervention in the community to bring forth hope. In addition, more community platform to encourage participation and positive sharing to counter the general reluctance to speak about cancer diagnosis, its treatment and care (because of fear ) can address the rising cancer stigma which hinders people from going for early detection. When society refused to talk about cancer, it prolonged the prevailing mystery about the condition, and also, strengthened the entrenched stigma of a cancer diagnosis. Active and activated survivors can help shift the perception of cancer as a fatal condition to a less stigmatised chronic condition [4].

All these elements can be integrated in the MOPCS framework to encapsulate the holistic occupational perspective approach within the ICF basic framework. Thus, the occupational model of care for cancer survivorship (MOPCS, Figure 1) provides better engagement of occupational participation with a broader and better target towards 'health-after-cancer' status. Clinicians and researchers in the field of occupational science can use this model to inform their direct interdisciplinary interventions with key health providers in occupational survivorship practices. The MOPCS model highlight the essential concept of occupational participation, and inform the research conducted by occupational therapists / scientists, providing a direction to therapists, oncology collaborators and health care providers such as the oncologists, primary care physicians, physical therapists, psychologists and counsellors. The key difference of MOPCS model and other cancer survivorship model is that MOPCS viewed survivors as 'active' (not passive recipient of more care) person - one who is informed and encourage/supported with self efficacy and received some self management support to be an active patient self-manager who actively persevere adapt and readjust towards the right balance and, in participating across key tasks like surveillance, health promotion and cancer prevention activities engagements. It also allows them to be better advocator model for cancer control in the society.

\section{Summary}

As a chronic disease, the after-effects of many cancer sites can be extensive, and tremendously varied from one person to another. Therapeutic health implications are beyond merely treating the illness, with a need to enable empowerment of patient self-management and self-care. The need to integrate the unique non-medical psychosocial and occupational needs of cancer survivors calls for direct occupational therapy to proactively assess and manage these issues in partnership with an informed patient. Having an occupational model like the MOPCS provides a conceptual guide to direct related occupational research and occupational clinical practices. An occupational perspective allows therapy professionals to better manage cancer conditions as a chronic condition that can lasts for years or decades. Most importantly, it promotes a healthy relationship that emphasise patients self management and where patients is an active advocate of his/her health (vs a passive recipient of prolonged care).

\section{Competing Interests}

The author declare that there is no competing interests regarding the publication of this article.

\section{References}

1. Oeffinger $\mathrm{KC}$, Argenbright $\mathrm{KE}$, Levitt GA, McCabe MS, Anderson PR, et al. (2014) Models of cancer survivorship health care: moving forward. Am Soc Clin Oncol Educ Book.

2. Loonen J, Blijlevens NM, Prins J, Dona DJ, Den Hartogh J, et al. (2018) Cancer Survivorship Care: Person Centered Care in a Multidisciplinary Shared Care Model. Int J Integr Care 18: 4.

3. SY Loh \& H Jonsson (2016) Cancer Survivorship Care: A perspective from an Occupational-Participation Approach. J Cancer Sci Ther 8: 179-184.

4. Loh SY (2020) Reducing Stigma of Cancer in Asia-An Important Strategy for Cancer Control. Journal of Oncology Research Review \& Reports. 